\title{
A Reservation Protocol Analysis for Multi-Channel Wireless Networks
}

\author{
Peristera A. Baziana, and Ioannis E. Pountourakis
}

\begin{abstract}
A synchronous multi-channel multi-access Medium Access Control (MAC) protocol for Wireless Local Area Networks (WLANs) is studied in this paper. The proposed protocol introduces an access control reservation scheme and requires a single radio per station. The receiver collisions phenomenon characterizes the performance of the proposed multi-channel system. A priority scheme is considered in order to primary serve the time-sensitive traffic such as voice, as compared to the delay tolerant data traffic. The innovation of this paper is the extensive and accurate study of the receiver collisions effect on the network performance in multi-traffic environment. An analytic discrete time Markovian model is developed for finite number of stations and channels. The performance measures of throughput, delay, and average rejection probability at destination are analytically estimated. Numerical results are presented for comparison for various numbers of channels and stations. The proposed MAC protocol provides a substantial contribution to the understanding of wireless multi-channel multi-traffic environments.
\end{abstract}

Index terms: wireless networks, multi-channel network, receiver collisions, multiple traffic, Markovian process, rejection probability

\section{INTRODUCTION}

Recent studies in Wireless Local Area Networks (WLANs) has introduced a variety of alternative communication protocols to improve network performance and guarantee a predefined Quality of Service (QoS) for diverse types of wireless applications, such as medical or military services. The latest research evolutions have expanded the use of wireless networks from conventional data communication to more complex communication modes including both data and real-time traffic. Special interest is spent to the adoption of specific Medium Access Control (MAC) protocols suitable for wireless environments, that could serve the different types of communication traffic considering the time-dependence and the priorities of real-time traffic [1]-[2].

In many studies for wireless networks, significant performance improvement is achieved by utilizing multiple channels instead of a single common shared one [3]-[5]. The use of multiple channels that do not interfere with each other offers many advantages, such as:

Manuscript received May 14, 2005; revised February 24, 2006, and January 11,2007 . The paper was presented in part at the Conference on Software, Telecommunications and Computer Networks (SoftCOM) 2004.

P. A. Baziana and I. E. Pountourakis are both with School of Electrical and Computer Engineering, National Technical University of Athens, 15773 Zografou, Athens, Greece, (e-mail: baziana@central.ntua.gr)
1. significant throughput amelioration, as multiple successful transmissions may occur simultaneously over different channels.

2. high load tolerance without performance degradation, which allows the growth of the system population.

3. high reliability and fault tolerance, as the mechanism used for collision resolution works independently of the reason of the unsuccessful transmission (collision or disruption of the channel).

4.extension of the useful life of existing systems, as the gradual growth of the system can be achieved by adding new channels depending on the load demands.

5. support of different levels of QoS, for diverse traffic types (voice or data).

In order to exploit the aforementioned advantages of the multi-channel scheme in wireless networks, special MAC protocols is required to be addressed. For example, the IEEE 802.11 specification does not exploit the above mentioned merits although the utilization of multiple channels is introduced at the physical layer, because the used MAC protocol is designed for a single-channel network [6]-[7]. In order to overcome this limitation, in latest studies some multichannel MAC protocols suitable for wireless communication have been proposed [4]-[5], [7]-[9]. These protocols are classified into four categories, according to the control mechanism that the competing stations follow to gain access to the network [9]:

a) the Dedicated Control Channel protocols: the stations have two radios, one specifically dedicated to monitor the control channel and the other for actual data communication.

b) the Common Hopping protocols: the stations have one radio and they hop together quickly among the channels until they agree for data transmission.

c) the Split Phase protocols: the stations have one radio and they periodically monitor a specified control channel.

d) the Multiple Rendezvous protocols: the stations have one or more radios to exchange control and data information over multiple control channels.

Although the requirement of only one radio in the common hopping and split phase protocols appears to be a significant advantage, other performance parameters should be taken into account too, such as the number of channels, the traffic priorities etc. Key role in the network performance plays the radio switching time. Its effect is critical in common hopping and multiple rendezvous protocols as they are based on hopping techniques and they incur a penalty at every hopping. 
In the contrary, in split phase protocol the radio switching time slightly influences the network performance [9].

In general, there are two major causes of packet loss in multi-channel networks [11]-[12]. First packets are destroyed if two or more stations transmit their packets in the same channel of the multi-channel system and they are overlapped in time. This phenomenon is referred as channel collision. Second additional packets are aborted, when a collision-free packet transmission cannot be picked up by the intended destination since the receiver may be tuned to another channel for receiving a packet from another source. This phenomenon is called receiver collision [10].

Although the receiver collisions significantly affect the performance of a wireless multi-channel network, its influence is more critical in multi-traffic environment due to the different priorities and QoS demands of real-time and data traffic. In [9], the multiple rendezvous Multi-Channel MAC (McMAC) protocol is presented and the throughput is given for finite number of channels and stations based on a Markovian model and assuming slotted Aloha access scheme. The packet loss at destination is introduced without any consideration for time-sensitive traffic types, such as voice. In [1]-[2], two different traffic types are studied in a multichannel architecture environment providing different number of channels for each traffic category to access the system. The paper evaluates the network performance using Markovian analysis without considering the receiver collisions phenomenon.

In this study we suggest a synchronous transmission reservation $\mathrm{MAC}$ protocol for a wireless multi-channel network using a single radio per station. The time axis is dividing into frames of equal length. Each frame is splitted into two phases: the control phase that is used as the reservation part of the protocol and the transmission phase for the real information transmission, like in the split phase protocols. We evaluate the performance measures for the two traffic types (voice and data) using different priorities, avoiding destination conflicts according to a proposed arbitration rule which takes into account the real time transmission attribute or not for each traffic category. The rigorous and detailed analysis is based on a Markovian process. The structure of the control packet joints with the arbitration rule for destination conflicts avoidance. The following receiver collisions analysis constitutes the important part of this study.

This paper is organized as follows. The network model and the assumptions of the analysis are described in Section 2. In Section 3 the model analysis is extensively described and the performance measures are derived. Comparative numerical results are discussed in Section 4. Concluding remarks are outlined in Section 5.

\section{NETWORK MODEL AND ASSUMPTIONS}

A multi-channel multi-access wireless LAN of $N$ parallel bidirectional channels all of the same bandwidth in $\mathrm{Hz}$ is considered, like in [1]-[2]. A finite number, $M$, of stations that have access to each channel of the system is assumed. All stations are within each other's transmission range, so as a transmission reaches its destination in a single hop. The multichannel network architecture is similar to this of [2].

Network traffic is classified into two types: real-time traffic such as voice, and data traffic such as file transfer. We assume that each station can generate both voice and data traffic. The transmission time of a fixed size control packet is used as time unit and is called minislot. The voice or data (voice/data, V/D) packet transmission time normalized in minislot time units is $L$ $(L>1)$ and is called V/D slot. A control packet consists of the following information about the V/D packet transmission: 1) the source address, 2) the destination address, 3) the packet generation time and 4) the type of traffic (V/D), as Fig. 1 shows. All channels use the same time reference which we call frame. Each frame consists of two contiguous time phases: the control phase and the transmission phase, as Fig. 1 illustrates. During the control phase, all stations meet on a default channel, let be channel-1, to gain access to the multichannel network for V/D packet transmission and to be informed for possible transmissions towards them. The control phase consists of $N$ contiguous minislots that have one-to-one correspondence to the $N$ channels, i.e. minislot- 1 corresponds to channel-1, minislot-2 corresponds to channel-2, etc. During the transmission phase, the V/D packets transmissions occur over the $N$ channels. We define the duration of a frame as the time interval that includes $N$ minislots for control packets transmissions (control phase) followed by the V/D packet transmission time $L$ (transmission phase). Thus, the frame time duration is $F=N+L$ time units. The existence of a common clock to all stations is assumed. Time axis is divided into contiguous frames of equal length $F$ and these frames are synchronized across all channels. Irrespectively from the packet type, each station has access to all channels, i.e. it can transmit and/or receive constant length V/D packets that fit to V/D slot size.

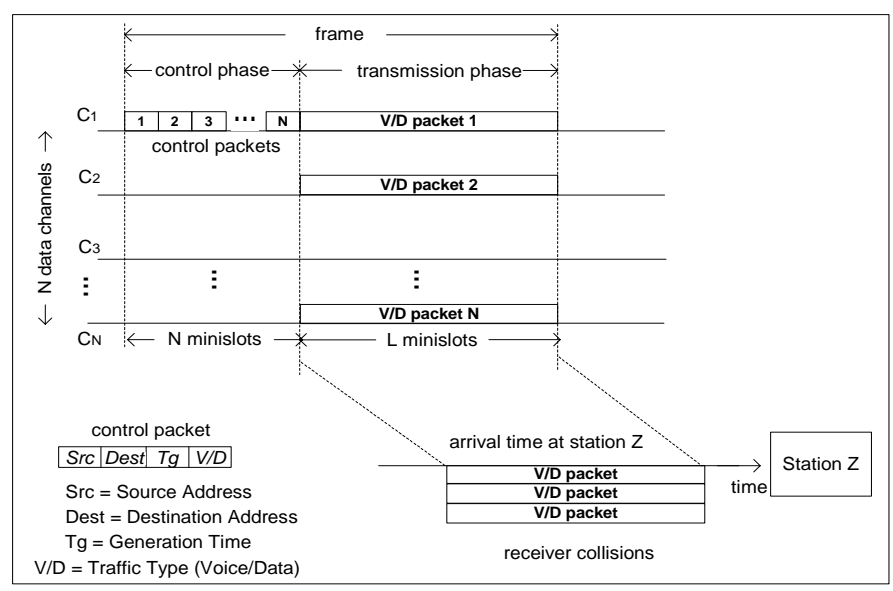

Fig. 1. Network model: Access and reception mode.

Each station is equipped with a receiver buffer and a transmitter buffer, each one with capacity of one packet. If the transmitter buffer is empty the station is said to be free, otherwise it is backlogged. V/D packets are generated independently at each station following a geometric distribution, i.e a V/D packet is generated at each frame with probability $p$. A backlogged station retransmits the 
unsuccessfully transmitted V/D packets following a geometric distribution with probability $p_{1}$ per frame and defers the transmission by one frame with probability $\left(1-p_{1}\right)$. If a station is backlogged and generates a new packet, the packet is lost and never returns.

In our analysis, the propagation delay is considered to be small enough so that the transmitting station is informed until the end of the frame whether the transmission was successful or not. Also, the radio switching time is assumed to be negligible according to the results of [9]. Neither fading phenomena nor channel interference are considered.

According to the access mode, if a station has to send a V/D packet to another during a frame, first it chooses randomly one among the $N$ channels for the packet transmission. Each channel is chosen with equal and constant probability $P_{i}=1 / N$. Then, during the control phase of the frame it sends a control packet over the channel-1 at the control minislot that corresponds to the selected transmission channel. The transmitted control packets compete according to the slotted Aloha protocol to gain access to each channel. Thus, if more than one stations select the same channel to (re)transmit, a collision at the corresponding control minislot will occur. Only the successful transmissions over the $N$ minislots are allowed to be transmitted during the transmission phase of the frame.

According to the reception mode, it is considered that each destination is randomly selected with equal probability $1 / M$ (for the sake of generality we suppose that a station may send to and receive from itself), like in [11]-[12]. If two or more $\mathrm{V} / \mathrm{D}$ packets from different channels are addressed to the same destination, one of them is correctly received according to specified arbitration priority rules and the others are aborted. This phenomenon is called receiver collision [9]-[11]. Especially, if one voice packet and one data packet are destined to the same destination, the destination receives the voice packet and rejects the data one providing high priority to the time-sensitive voice traffic. In addition, if two or more voice packets are destined for the same station, the destination receives the eldest one and aborts the others. The same criterion is applied if two or more data packets are destined to the same destination.

A backlogged station is getting free at the end of a frame if it manages to be retransmitted without collision over a control minislot and is received at destination without receiver collision. A free station becomes backlogged in case of an unsuccessful control packet transmission or receiver collision.

\section{MODEL ANALYSIS}

The performance of the examined system can be described by a discrete time Markov chain. We denote the state of the examined system by $\left\{X_{t}, t=0,1,2, \ldots\right\}$, where $X_{t}=0,1, \ldots M$ is the number of backlogged stations at the beginning of a frame. Let:

$H_{t}=$ The number of new V/D packets arrivals during a frame, $t=0,1,2, \ldots$

$A_{t}=$ The number of correctly received V/D packets at the end of a frame, $t=0,1,2, \ldots$
$S_{k}=$ The number of successful control packets (re)transmissions, conditional that $k$ free and/or backlogged stations attempt transmission during a frame and $0 \leq S_{k} \leq \min (N, k)$.

$A_{n}=$ The number of correctly received V/D packets at destination, conditional that $n$ successful control packet (re)transmissions occurred during a frame, $S_{k}=n$ for every $S_{k}>0$.

The probability $\operatorname{Pr}\left[S_{k}=n\right]$ of $n$ successes from $k$ control packets (re)transmissions during a frame is given in [11]-[12] and it is:

$\operatorname{Pr}\left[S_{k}=n\right]=\frac{(-1)^{n} N ! k !}{N^{k} n !} \sum_{j=n}^{\min (N, k)} \frac{(-1)^{j}(N-j)^{k-j}}{(j-n) !(N-j) !(k-j) !}(1)$

and $0 \leq n \leq \min (N, k)$.

The probability $\operatorname{Pr}\left[A_{n}=r\right]$ of $r$ correctly received V/D packets at destination, given that $n$ control packets are successfully (re)transmitted during a frame is given in [9]. It is:

$$
\operatorname{Pr}[A(n)=r]=\left(\begin{array}{l}
M \\
r
\end{array}\right) \sum_{i=0}^{r}(-1)^{i}\left(\begin{array}{l}
r \\
i
\end{array}\right)\left(\frac{r-i}{M}\right)^{n}
$$

and $1 \leq r \leq \min (n, N)$ for every $n \geq 1$.

We define the function $\Phi(x, y, z)$ as the product of the probability of $y$ successes from $x$ free and/or backlogged control packets (re)transmissions over the $N$ control minislots, times the probability of $z$ correctly received V/D packets at destination during a frame, i.e.:

$$
\Phi(x, y, z)=\operatorname{Pr}\left[S_{x}=y\right] \operatorname{Pr}\left[A_{y}=z\right]
$$

Also, we define the conditional probabilities [11]-[12]:

1. $q_{\text {in }}$ that $i$ out of $n$ backlogged stations attempt to transmit with probability $p_{1}$ during the frame. It is given by:

$$
q_{n, i}=\left(\begin{array}{l}
i \\
n
\end{array}\right) p_{1}^{n}\left(1-p_{1}\right)^{i-n}, \quad i \geq n
$$

2. $Q_{\text {in }}$ that $i$ out of $(M-n)$ free stations attempt to transmit with probability $p$ during the frame. It is given by:

$$
Q_{n, i}=\left(\begin{array}{c}
M-i \\
n
\end{array}\right) p^{n}(1-p)^{M-i-n}, \quad M-i \geq n
$$

The Markov chain $\left\{X_{t}, t=0,1,2, \ldots\right\}$ is homogeneous, aperiodic, and irreducible. The one step transition probabilities are given by: $P_{i j}=\left(X_{t+1}=j \mid X_{t}=i\right)$, where:

Case A: $j<i-N$ then:

$$
P_{i j}=0
$$

Case B: $j=i-N$ then:

$$
P_{i j}=Q_{0, i} q_{N, i} \Phi(N, N, N)
$$

Case C: $i-N<j<i$ then: 


$$
\begin{aligned}
& \left\{\begin{aligned}
\sum_{n=i-j}^{N} q_{n, i} & \sum_{m=0}^{\min (M-i, N-n)} Q_{m, i} \\
& \times \sum_{s=0}^{n+j-i} \Phi(n+m, i-j+m+s, m+i-j)+
\end{aligned}\right. \\
& P_{i j}=\left\{\begin{array}{c}
+\sum_{n=i-j+2}^{N} q_{n, i} \sum_{m=N-n+1}^{\min (M-i, N+j-i-1)} Q_{m, i} \\
\times \sum_{s=0}^{N-m+j-i-1} \Phi(n+m, i-j+m+s, m+i-j)+
\end{array}\right. \\
& \begin{aligned}
+\sum_{n=N+1}^{i} q_{n, i} & \sum_{m=0}^{\min (M-i, N+j-i-1)} Q_{m, i} \\
& \times \sum_{s=0}^{N-m+j-i-1} \Phi(n+m, i-j+m+s, m+i-j)
\end{aligned}
\end{aligned}
$$

Case D: $j=i$ then:

$$
P_{i j}=\left\{\begin{array}{l}
Q_{0, i} \sum_{n=2}^{i} q_{n, i} \Phi(n, 0,0)+q_{0, i} \sum_{m=0}^{N} Q_{m, i} \Phi(m, m, m)+ \\
+\sum_{m=1}^{m i n(M-i, N-1)} Q_{m, i} \sum_{n=1}^{N-m} q_{n, i} \sum_{s=0}^{N-m} \Phi(n+m, m+s, m)+ \\
+\sum_{m=1}^{m i n} Q_{m, i}^{M-i, N-1)} \sum_{n=N-m+1}^{i} q_{n, i} \sum_{s=0}^{N-m-1} \Phi(n+m, m+s, m)
\end{array}\right.
$$

Case E: $j>i$ then:

$$
P_{i j}=\left\{\begin{array}{c}
Q_{j-i, i} \sum_{n=0}^{i} q_{n, i} \Phi(j-i+n, 0,0)+ \\
+\sum_{m=1}^{m i n(} Q_{j-i+m, i} \sum_{n=0}^{N-j, N-j+i)} q_{n, i} \\
\quad \times \sum_{s=0}^{N-m} \Phi(j-i+n+m, m+s, m)+ \\
m i n(M-j, N-1) \\
\sum_{m=1}^{N} Q_{j-i+m, i} \sum_{n=N-m-j+i+1}^{i} q_{n, i} \\
\times \sum_{s=0}^{N-m-1} \Phi(j-i+n+m, m+s, m)
\end{array}\right.
$$

\section{A. Performance Measures}

Since the Markov chain $\left\{X_{t}, t=0,1,2, \ldots\right\}$ is ergodic, the steady state probabilities $\boldsymbol{\pi}_{i}, i=0,1, \ldots M$, can be estimated by solving the system of the following linear equations:

$$
\pi=\pi P
$$

and: $\quad \sum_{i=0}^{M} \pi_{i}=1$

where: $\boldsymbol{P}$ is the transition matrix with elements the probabilities $P_{i j}$ and $\pi$ is a row vector with elements the steady state probabilities $\boldsymbol{\pi}_{\boldsymbol{i}}$.

The conditional throughput $S_{r c}(i)$ is the expected value of the output rate during a frame given that the number of the backlogged stations at the beginning of the frame is $i$, i.e. $S_{r c}(i)=E\left\{A_{t} \mid X_{k}=i\right.$ \}and is given by: $S_{r c}(i)=$

$$
\begin{aligned}
& =\left\{\begin{array}{l}
\sum_{k=1}^{N} k \sum_{m=0}^{m i n} Q_{m, i} \sum_{\substack{n=0 \\
m+n \geq k}}^{m-i, N)} q_{n, i} \sum_{s=0}^{\min (N-m, i)} \Phi(n+m, k+s, k)+ \\
+\sum_{k=1}^{N-1} k \sum_{m=0}^{\min (M-i, N)} Q_{m, i} \sum_{n=N+1}^{i} q_{n, i} \sum_{s=0}^{N-k-1} \Phi(n+m, k+s, k)+
\end{array}\right. \\
& +\sum_{k=1}^{N-1} k \sum_{m=N+1}^{M-i} Q_{m, i} \sum_{n=0}^{i} q_{n, i} \sum_{s=0}^{N-k-1} \Phi(n+m, k+s, k)
\end{aligned}
$$

Thus, the steady state average throughput $S_{r c}$ is given by:

$$
S_{r c}=\frac{L}{F} E\left[S_{r c}(i)\right]=\frac{L}{F} \sum_{i=0}^{M} S_{r c}(i) \pi_{i}
$$

The steady state average number $B$ of backlogged stations is given by:

$$
B=E(i)=\sum_{i=0}^{M} i \pi_{i}
$$

The conditional input rate $S_{i n}(i)$ is the expected number of arrivals during a frame given that the number of the backlogged stations at the beginning of the frame is $i$, and is given by:

$$
S_{\text {in }}(i)=E\left[H_{t} \mid X_{t}=i\right]=(M-i) p
$$

The steady state average input rate $S_{\text {in }}$ is given by:

$$
S_{\text {in }}=\sum_{i=0}^{M} p(M-i) \pi_{i}
$$

The delay $D$ is defined as the average number of frames that a V/D packet has to wait until its successful transmission. Delay is calculated through Little's formula:

$$
D=1+\frac{B}{\operatorname{Sin}}
$$

Also, we define the average rejection probability $P_{\text {rej }}$ of a packet at destination in steady state as the ratio of the average number of packets rejected due to receiver collisions, to the average number of successfully transmitted control packets over the control minislots during a frame, i.e.: 


$$
P_{r e j}=\frac{S-S_{r c}}{S}
$$

where: $S$ is the expected value of the successes during the control phase of a frame in steady state.

In order to quantify the receiver collision impact, we choose for evaluation the points that correspond to $S(\max )$ and $S_{r c}(\max )$. So, we define:

$$
P_{r e j}(\max )=\frac{S(\max )-S_{r c}(\max )}{S(\max )}
$$

where: $S(\max )$ is the maximum value of the successes during the control phase of a frame in steady state and $S_{r c}(\max )$ is the maximum value of $S_{r c}$ during a frame.

\section{NUMERICAL RESULTS}

The numerical solution of the proposed Markovian queuing model is presented in this section. In order to verify the accuracy of the theoretical analysis, we developed a specific network simulator based on the $\mathrm{C}$ programming to simulate the proposed system performance. The developed simulator implements an extensive discrete-event simulation model and uses as confidence level $(1-\alpha)$ the value $99 \%$ that gives almost one to one correspondence to the actual system, as Fig. 3 and 4 present. The performance measures for various system population and diverse numbers of channels are illustrated in Fig. 2-7. The retransmission probability $p_{I}$ is common for all busy stations.

Fig. 2 and 3 illustrate the throughput $S_{r c}$ curves versus the birth probability $p$ for different number of channels $N$ and stations $M$. In order to evaluate the receiver collisions effect on the network throughput, Fig. 2 and 3 depict the expected value $S$ of the control packets successes during a frame in the multi-channel system.

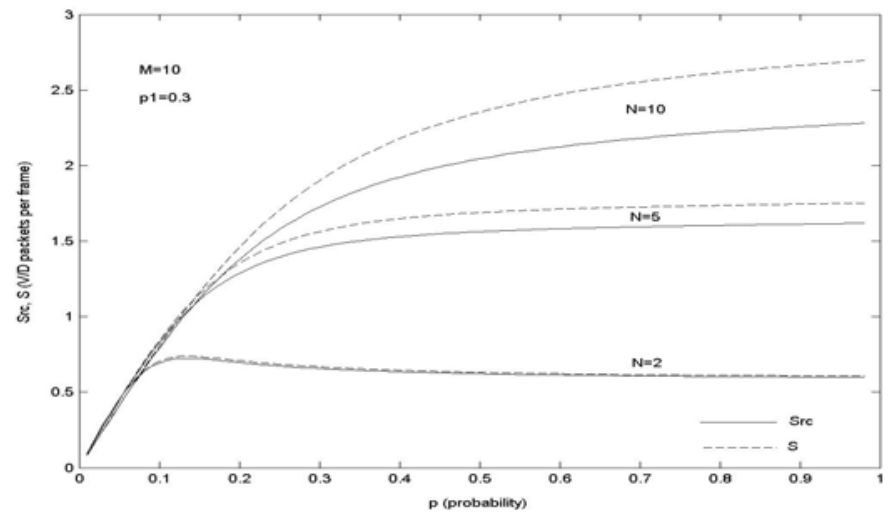

Fig. 2. Throughput $S_{r c}$ and expected value $S$ versus probability $p$ of V/D packets generation per frame, for $M=10$ stations, $N=2,5,10$ channels and $p_{I}=0.3$.

As Fig. 2 shows, the receiver collisions significantly affect on the throughput causing essential performance reduction. The packet loss at destination due to the receiver collisions is more noticeable at heavy load, due to the large number of backlogged stations, as it can be observed from Fig. 4.
Especially from Fig. 2 can be observed that for fixed $M$ and for a given value of birth probability the throughput reduction due to receiver conflicts is getting higher as $N$ increases. The explanation is that as $N$ grows, control packets collisions are reduced increasing on the other hand the expected value $S$ of the successes during the control phase of a frame and giving rise to receiver collisions. For example let $p=0.9$ and $M=10$, the $S_{r c}$ is reduced to: $1.3 \%$ for $N=2,7.58 \%$ for $N=5$, and $15.25 \%$ for $N=10$.

In the opposite, from Fig. 3 we observe the reverse behaviour. In other words, the throughput reduction due to receiver conflicts is getting lower as $M$ increases. The reason is that as $M$ grows for fixed $N$ and $p$, the offered load increases so control packets collisions also increase. This fact causes reduction of the expected value $S$ of the successes during the control phase and consequent reduction of the rate of receiver collisions. For example let $p=0.9$, the $S_{r c}$ is reduced to: $15.25 \%$ for $M=10,8.1 \%$ for $M=20$ and $5.4 \%$ for $M=30$.

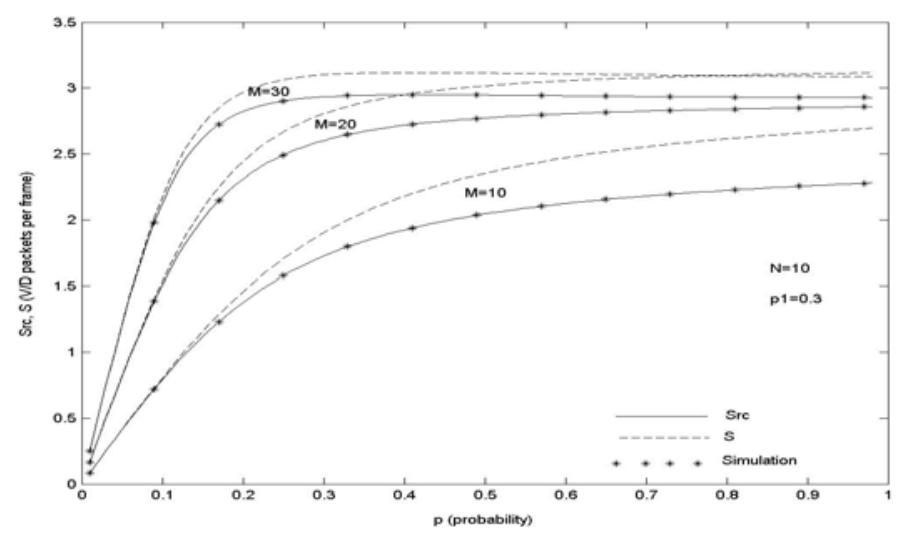

Fig. 3. Throughput $S_{r c}$ and expected value $S$ versus birth probability $p$ of V/D packets generation per frame, for $M=10,20,30$ stations, $N=10$ channels and $p_{l}=0.3$.

It is obvious that as $M$ grows control packets collisions increase and throughput is a decreasing function of $M$, for all $N$. The role of control packets collisions is more substantial as $N$ decreases with simultaneous growth of $M$. On the other hand, as $M$ and $N$ increase simultaneously we get more efficient use of the multi-channel network infrastructure. This is a critical point which justifies the receiver collision analysis.

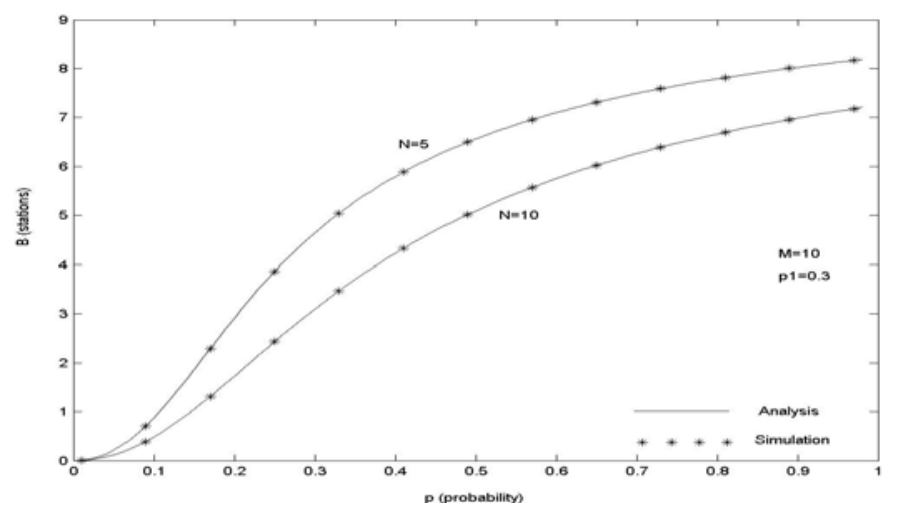

Fig. 4. Backlog $B$ versus probability $p$ of $\mathrm{V} / \mathrm{D}$ packets generation per frame, for $M=10$ stations, $N=5,10$ channels and $p_{l}=0.3$. 
Fig. 4 illustrates $B$ versus birth probability $p$ for $M=10$ and $N=5,10$. The numerical results provided by the simulation are also given in this figure. The figure verifies the behaviour of the system justifying the results of the previous two figures. In other words, the number of backlogged stations $B$ is a decreasing function of $N$ for fixed $M$, due to the rise of receiver collision effect. For example let $p=0.9$, for $N=1$ we get $B=9.87$, for $N=5$ we get $B=8.03$, and for $N=10$ we get $B=6.99$.

Fig. 5 presents the average percent rejection probability $P_{r e j}$ versus birth probability $p$ for $M=10$ and $N=2,5,10$. As it is expected from the above comments, the $P_{r e j}$ is an increasing function of $N$. This is noticed because as the number of channels $N$ increases, the probability of a control packet collision decreases. In this case, the number of successfully transmitted packets that reach their destination increases giving rise to the receiver collisions.

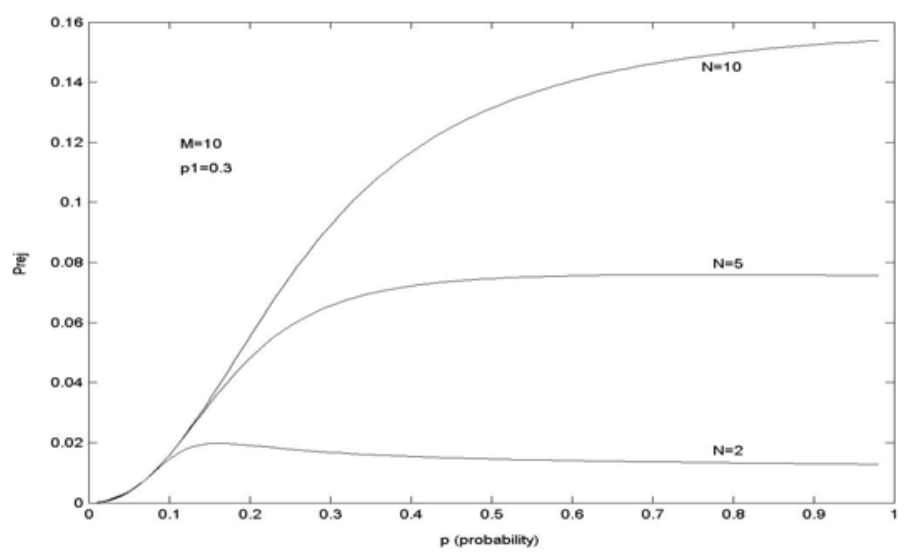

Fig. 5. Average Percent Rejection Probability $P_{r e j}$ versus probability $p$ of V/D packets generation per frame, for $M=10$ stations, $N=2,5,10$ channels and $p_{I}=0.3$.

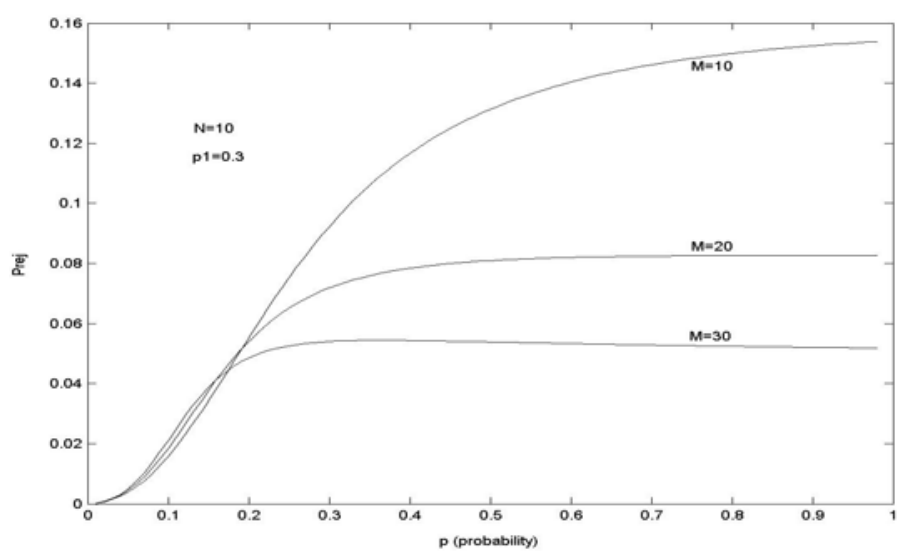

Fig. 6. Average Percent Rejection Probability $P_{r e j}$ versus probability $p$ of V/D packets generation per frame, for $N=10$ channels, $M=10,20,30$ stations and $p_{l}=0.3$.

It is evident that the relation between $N$ and $M$ determines the efficiency of the proposed multi-channel system. In other words the number of $N$ is related with control packet collision and the combination of $N$ with $M$ with the receiver collision phenomena. Another serious problem is the channel utilization which associates $N$ with $M$ as Fig. 3 shows. In a real analysis is necessary to take into account the correlation of these parameters in conjunction with the estimation of the maximum rejection probability $\operatorname{Prej}(\max )$ for the determination of the utilization criteria of the multi-channel system.

These performance limitations provided by Prej(max) are presented in Fig. 7. As it is depicted, the effect of receiver collisions in networks with fixed number of channels $N$ is more noticeable when the network occupies small number of stations $M$. As expected, Prej(max) is a decreasing function of $M$ for fixed $N$. It is obvious that as $M$ increases control packets collisions grow and the effect of destination conflicts is getting lower. Also, Prej(max) is an increasing function of $N$ for fixed $M$, as Fig. 7 shows. The explanation is that as $N$ grows control packets collisions decrease with simultaneous increase of receiver collisions.

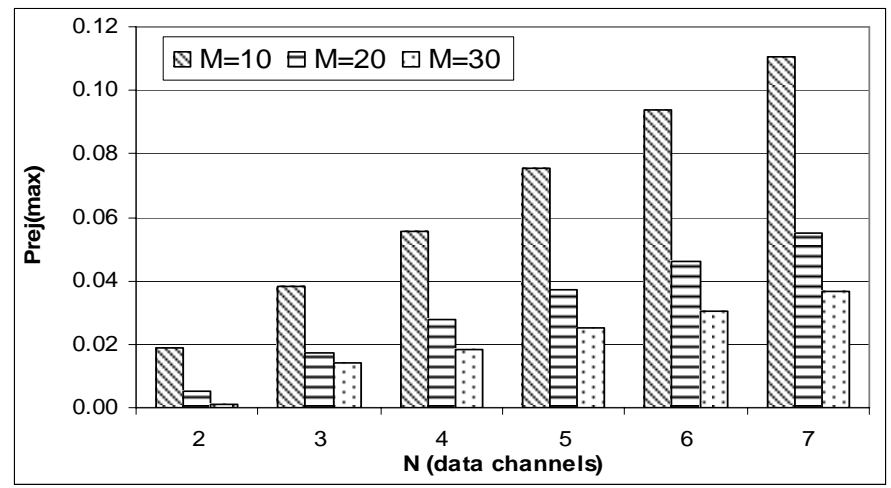

Fig. 7. Maximum Rejection Probability Prej(max) for $M=10,20,30$ stations and $N=2,3,4,5,6,7$ channels.

Fig. 8 plots the delay $D$ versus $S_{r c}$ for $M=10$ stations and $N=2,5,10$ channels. As it is shown, the slop of the curves at high values of $S_{r c}$ decreases as the number of channels $N$ increases, which gives robustness in the stability and manoeuvrability to load demands.

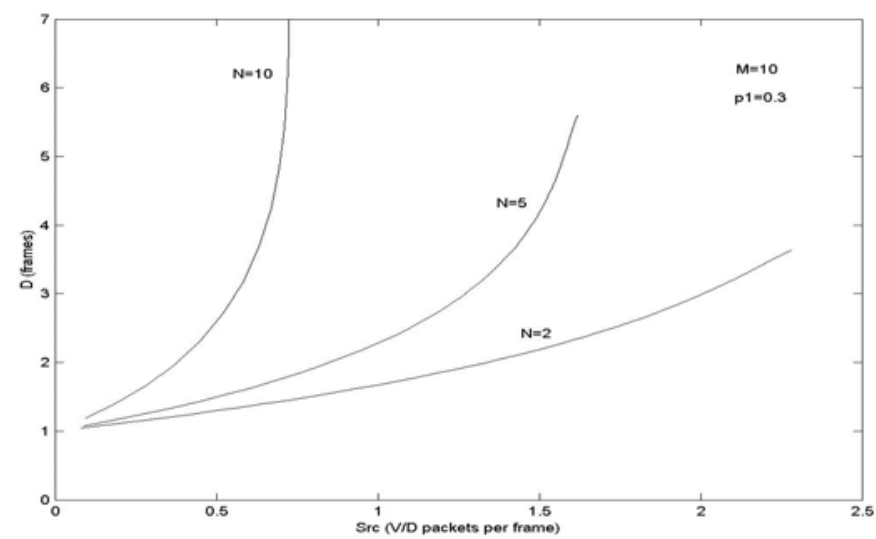

Fig. 8. Delay $D$ versus throughput $S_{r c}$ for $M=10$ stations, $N=2,5,10$ channels and $p_{1}=0.3$.

\section{CONCLUSION}

This paper presents a multi-channel MAC protocol for wireless networks with a single radio per station where both 
voice and data traffic is present. We introduce a synchronous reservation based access scheme in which each frame is splitted into two phases: the control phase that is used as the reservation part of the protocol and the transmission phase for the real information transmission, like in the split phase protocols. The receiver collisions that characterize the multichannel system are extensively examined and its effect on the network performance is studied. In our analysis, two levels of traffic priorities are considered in order to satisfy the different QoS demands of voice and data traffic. Thus, a priority scheme is assumed to primary serve the time-sensitive voice traffic as compared to the delay tolerant data traffic. The motivation of this paper is the extensive and accurate study of the receiver collisions in multi-traffic environment by means of a rigorous discrete-time Markovian process. The numerical results prove that the network performance essentially depends on the Prej(max) parameter. It was shown that for large population systems the effect of receiver collision is slight and can be ignored with only a small loss of accuracy. In the opposite case, in smaller systems the influence of receiver collisions pays a significant role to the network performance and cannot be neglected. Finally, this paper makes a significant contribution to the understanding of multichannel multi-traffic wireless networks performance analysis and offers additional insights.

\section{REFERENCES}

[1] W.Yue, Y.Matsumoto: Exact evaluation of multi-traffic for wireless PCS networks with multi-channel, Lecture Notes in Computer Science, Vol. 1970, pp. 427-438, 2000.

[2] W.Yue, Y.Matsumoto: Exact Analysis of Multi-Traffic Wireless Communication Networks with Reserved and Nonreserved Multi-Channel, IEICE Trans on Communications, Vol. E84-B, No. 4, pp. 786-794, 2001.

[3] S.Kumar, V.S.Raghavan, J. Deng: Medium Access Control Protocols for Ad-Hoc Wireless Networks: A Survey, Elsevier Ad Hoc Networks Journal, 2004.

[4] Y.S.Han, J.Deng, Z.J.Haas: Analyzing Multi-Channel Medium Access Control Schemes with ALOHA Reservation, IEEE Trans on Wireless Communications, to appear.

[5] A.Chockalingam, W.Xu, M Zorzi, L.B.Milstein: ThroughputDelay Analysis of a Multichannel Wireless Access Protocol, IEEE Trans on Vehicular Technology, Vol. 49, No. 2, pp. 661671, March 2000.

[6] IEEE 802.11 Working Group: Wireless LAN Medium Access Control (MAC) and Physical Layer (PHY) specifications, 1997.

[7] J.So, N.Vaidya: Multi-Channel MAC for Ad Hoc Networks: Handling Multi-Channel Hidden Terminals Using A Single Tranceiver, in Proc. of the ACM MOBIHOC, 2004.

[8] H.W.So, J.Walrand: McMAC: A Multi-Channel MAC Proposal for Ad-Hoc Wireless Networks, Technical Report, Apr. 2005.

[9] J.Mo, H.W.So, J.Walrand: Comparison of Multi-Channel MAC Protocols, in Proc. of the 8-th ACM/IEEE International Symposium on Modeling, Analysis and Simulation of Wireless and Mobile Systems, pp. 209-218, Oct. 2005.

[10] I.E.Pountourakis: Performance Evaluation with Receiver Collisions Analysis in Very High-Speed Optical Fiber Local Area Networks Using Passive Star Topology, IEEE Journal of
Lightwave Technology, Vol.16 No.12, pp 2303-2310, Dec. 1998.

[11] P.A.Baziana, I.E.Pountourakis: Multi-channel MAC protocol: Performance mathematical analysis and comparison, in Proc. of SoftCOM 2004-12 $2^{\text {th }}$ International Conference on Software, Telecommunications and Computer Networks, pp. 146-150, 2004.

[12] I.E.Pountourakis, P.A.Baziana: Markovian receiver collision analysis of high-speed multi-channel networks, Mathematical Methods in the Applied Sciences Journal, Vol. 29, Issue 5, pp. 575-593, 2006, DOI: 10.1002/mma.701.

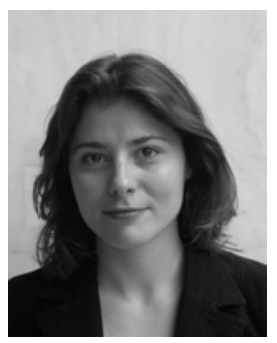

Peristera A. Baziana received the Diploma degree in Electrical and Computer Engineering from the University of Patras, Patras, Greece in 1998. From 1999 to 2002, she has participated in research programs of the Greek PTT Organization (O.T.E.) as a researcher of University of Patras. Since 2002 she has been working towards the Ph.D. degree at the Electronic and Information Engineering Division, School of Electrical and Computer Engineering of National Technical University of Athens, Athens, Greece at the field of architectures and protocols for optical networks. Her current research interests include optical communications, OBS networks, MAC protocols and queuing analysis. She is a member of the Technical Chamber of Greece.

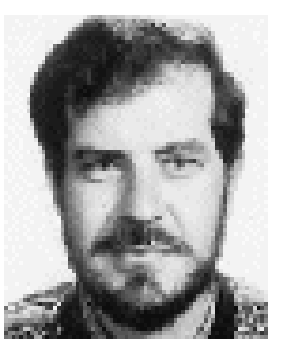

Ioannis E. Pountourakis is a Professor at the Communications, Electronic and Information Engineering Division, School of Electrical and Computer Engineering of National Technical University of Athens (N.T.U.A). His research interests include Optical Communication Networks, Network Architecture and Protocols, Performance Evaluation and Stability. He has taught several undergraduate and graduate courses at NTUA, supervised many doctoral students working in the areas of queuing analysis of contention resolution mechanisms in local area networks and optical networks, WDM network design, optical networks architectures, analysis of data link layer protocols, evaluation of performance of computer systems, etc and has been reviewer in International Journals, Conferences, and research project proposals. He has participated and organized many International Conferences. He has also participated in several RACE projects and in several national research programs dealing with communication networks. $\mathrm{He}$ is member of IEEE and the Greek society of Computer Science. 